\title{
An eHealth Platform for Instant Interaction among Health Professionals
}

\author{
CE Chronaki ${ }^{1}$, F Chiarugi ${ }^{1}$, E Mavrogiannaki ${ }^{1}$, C Demou ${ }^{1,2}$, P Lelis ${ }^{1,2}$, D Trypakis ${ }^{1}$, M Spanakis $^{1,2}$, \\ M Tsiknakis ${ }^{1}$, SC Orphanoudakis ${ }^{1,2}$ \\ ${ }^{1}$ Institute of Computer Science, FORTH, Heraklion, Crete, Greece \\ ${ }^{2}$ Computer Science Department, University of Crete, Heraklion, Crete, Greece
}

\begin{abstract}
In an age where medical collaboration is the key to effective healthcare delivery and continuity of care, the location of experts and the management of their time are affecting the wide deployment of eHealth services. In this setting, the use of instant messaging and presence technologies provides a possible solution. A collaboration platform based on instant messaging and presence technology has been employed to implement flexible and adaptable eHealth services. The platform is characterized by the use of medical device components that currently support real-time and/ or store-andforward access to spirometry examinations, medical imaging, vital signs, ECG, and auscultation data. Other important features of the platform are eHealth service profiles that entail electronic document templates conforming to HL7's clinical document architecture $(C D A)$, the electronic signing of clinical objects using smart cards, and the use of Object Management Group's $(O M G)$ person identification service (PIDS) to interoperate with clinical information systems.

So far, eHealth services profiles have been designed for cardiology and respiratory problems. The storage of clinical data in actively shared episode folders and the flexible messaging services that provide users with feedback, feedthrough, awareness, and notification irrespective of their location, have been immediately embraced by the healthcare community. In the immediate future, eHealth service profiles are expected to be further refined based on user feedback and usage data to support service evolution and thus, lead to even more effective service provision in healthcare.
\end{abstract}

\section{Introduction}

The two-year deployment of WebOnCOLL, a medical collaboration environment based on shared workspaces in HYGEIAnet, the regional health information network of Crete, has demonstrated that eHealth services offer tangible clinical, social, and economic benefits to all parties concerned [1]. Based on instant messaging technologies, which facilitate management of communication rather than information exchange and address the requirement for easy to use and accessible eHealth services in a regional or trans-regional setting, WebOnCOLL has evolved into an eHealth platform that supports an expanding list of medical devices as shown in figure 1 .

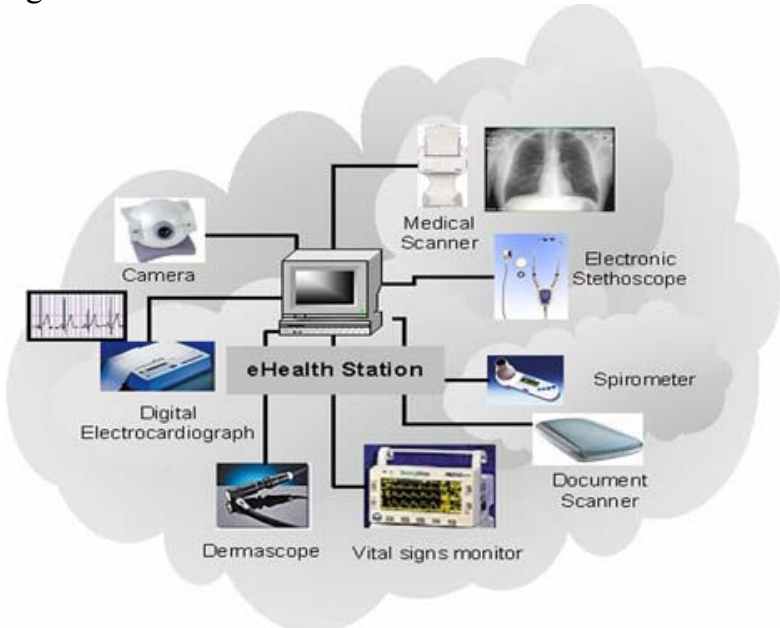

Figure 1. A wide variety of medical devices has been integrated to the eHealth station.

The backbone of WebOnCOLL is Jabber, a scalable open-source server implementing the IMPP XML protocol for instant messaging and presence [2]. To facilitate medical collaboration, Jabber was extended with a shared workspace component that supports subscription and notification on content updates. The messenger of the portal, now the centerpiece in medical collaboration, enables a smooth transition from asynchronous to synchronous interaction. Each shared workspace corresponds to a "chat room" where users interact by sharing multimedia clinical data relevant to a particular episode of care. Various medical device components have been developed and integrated in the eHealth platform complying with relevant interoperability standards. The integrated medical devices include a spirometer, ECG devices of different manufacturers, real-time vital signs monitor, real-time 12-lead ECG, and an electronic stethoscope. The corresponding components may be downloaded on demand and allow the sharing of clinical data produced 
by the corresponding devices in real-time or store-andforward operating mode. Interoperability with the clinical systems managing the electronic health record segments in primary healthcare provides access to local clinical data of the patient, while the Integrated Electronic Health Record service refers back to the patient's complete medical history.

Based on the suspected medical problem and the current clinical context, predefined document templates following the HL7/CDA ANSI standard are automatically filled out with relevant clinical data. These electronic documents are reviewed and digitally signed by the responsible physician. Smart cards and a regional Public Key Infrastructure are used to ensure authorization, confidentiality, integrity, and non-repudiation. Digital signatures of clinical items follow the W3C/ IETF standard for electronic signatures in XML [3].

The current version of the eHealth platform, with its new innovative features has been well-received by health professionals. In addition, the increased availability and accessibility of the service, as well as the flexible notification schemes it supports, are expected to further reduce the time required to bring medical expertise to the point of need.

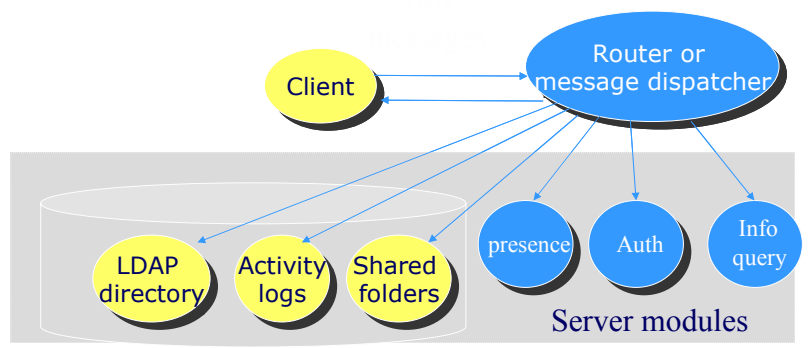

Figure 2. Structure of the IMPP backbone.

\section{Instant messaging \& presence}

Although teamwork has always been an important aspect of the medical practice, the proliferation of medical subspecialties and the availability of advanced diagnostic methods constitute medical collaboration now more significant than ever. Currently, the advance of health information networks and the Internet provides the necessary infrastructure for the evolution of clinically significant eHealth services. eHealth services address the rising demand for personal health management and the lack of specialized medical expertise at the point of need by facilitating the sharing of health data between the site of the patient, the family doctor, the pharmacy, and various healthcare facilities.

The eHealth platform builds on the popular metaphors of web portals and shared workspaces. Each workspace corresponds to an active episode folder. Medical professionals registered with the system have personal pages that provide access to episodes they created or have been invited in. Each episode folder includes shared multimedia clinical data and indication of availability for each of the participants. The environment provides instant messaging, presence, and notification services. A user may request to be notified when a particular episode folder changes i.e. when clinical data of a particular type become available, or when a specific expert navigates into the folder or looks at a particular item. Notification may be received through audio alerts, email messages, or SMS. The support of instant messages and awareness makes launching of audio-visual conferencing and the use of real-time diagnostic tools more convenient and user-friendly.

The overall architecture of the server is based on Jabber, an open source instant messaging server, which supports the IMPP protocol of IETF for instant messaging and presence [2]. This is an open extensible XML protocol that facilitates rapid prototyping using web technologies. The basic configuration of the jabber server has been extended with database support for the storage of clinical documents and accounting (see figure 2). To achieve this goal, additional XML namespaces have been introduced to allow not only the management of clinical folders and the clinical objects stored in them, but also notification events for their status.

\section{3. eHealth station}

A typical eHealth station located at a primary healthcare facility, a community office, or the home of a patient, may interface a wide range of medical devices and provide access to the medical collaboration environment via a portal like interface.

Consider the typical case of a General Practitioner (GP) or community doctor in a remote healthcare facility that faces a difficult episode or an emergency. Since the clinical findings raise suspicion of an acute condition, the GP requests assistance from a central hospital. The GP records the clinical findings in the electronic patient record (EHR) including a digital electrocardiogram and optionally laboratory data. Then, an appropriate eHealth profile (e.g. "cardiology/suspected AMI") is selected and relevant data are automatically retrieved from the EHR and included in an electronic document for second opinion request, tailored to the problem at hand. The GP reviews the consultation request maybe including additional information and digitally signs the document using a personal smart card.

Then the GP searches for an appropriate center or an available expert and sends an invitation to review the episode. The GP may request to be notified when an expert enters the episode folder or views a particular clinical item. In some cases, only asynchronous interaction is necessary: the expert views the data, requests some clarification or older ECGs, and files a diagnostic report.

Alternatively, the expert may consider the option of a televisit as in cases where auscultation or short-term real- 
time cardiac monitoring is considered necessary. Then, an appointment is scheduled. The patient is connected to the real-time device and his vital signs are acquired and displayed locally. When the expert goes on-line, a connection is established and the ECG data are shown synchronously on the remote screen. Both the expert and the GP may record snapshots of the cardiac activity in the episode folder. A visual conference may be also established. Following the synchronous collaboration session, the expert selects one of the available diagnostic report templates, fills out observations, remarks, and suggestions, and digitally signs it. The GP immediately views the report as part of the shared episode folder. The episode remains active until the GP files an outcome report, at which time the episode is archived.

\subsection{Medical device components}

A wide variety of medical devices have been integrated to the eHealth station (see Figure 3). Each device component has been wrapped up as one or more ocx controls, which can be embedded in a web page using javascript. These controls can be downloaded on demand from the Internet and configured in the context of different eHealth services.

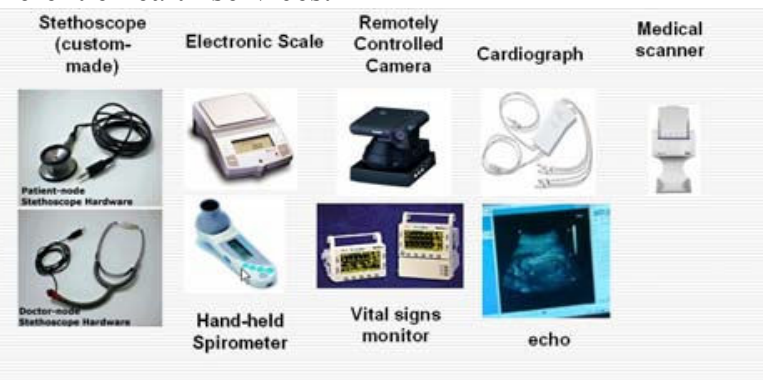

Figure 3. Medical devices currently integrated.

Currently, the medical devices that have been integrated include an electronic stethoscope, an electronic scale, a vital signs monitor, a hand-held spirometer, cardiographs of different brands, and various radiology modalities (see figure 3 ).

Some of these medical devices support wellestablished standards that facilitate integration. For instance, in the case of radiology modalities, the DICOM component developed at FORTH, can communicate with any DICOM-conformant radiology device or medical scanner to retrieve radiology examinations in the DICOM standard. Furthermore, appropriate software has been developed to convert digitalized diagnostic images or video into DICOM. DICOM viewing tools, in turn, can be used as helper applications to view the medical images or video.

In the case of electrocardiographs, there is a proliferation of standards some proprietary and some open (www.openecg.net). There is also an initiative embraced by FDA and HL7 for an XML-based file format that would allow the storage of annotated time series data. In Europe, the accepted standard is SCP-ECG and the eHealth station supports it. For electrocardiographs that do not support the standard, specialized software makes the appropriate conversion.

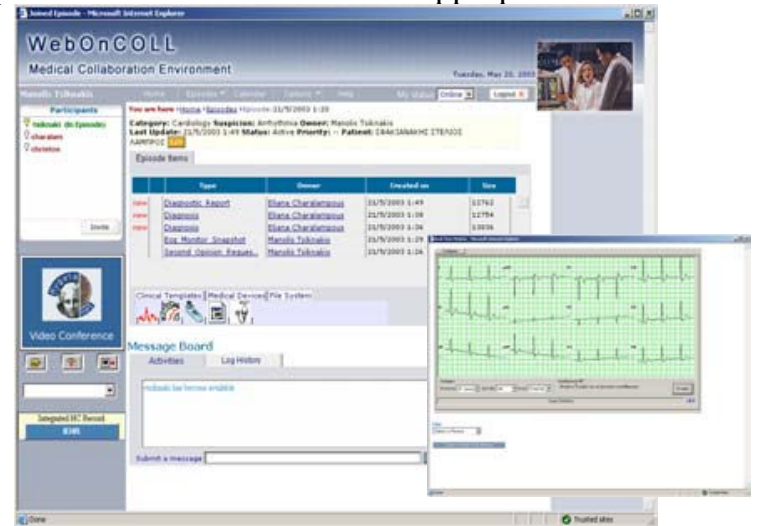

Figure 4. Interface of the eHealth station, when inviting an expert to receive a real-time 12-lead ECG.

An electrocardiograph that allows continuous ECG recording can be used both in store-and-forward and in real-time mode. In all cases, instant messaging and presence facilitates communication among the participants. The healthcare professional close to the patient prepares the patient and starts recording cardiac activity, while at the same time he/she is able to store 10 sec snapshots in the shared clinical folder according to the SCP-ECG standard. In the meantime, instant messaging and presence is used to notify an appropriate expert and invite him/her to join a real-time session (see figure 4). When the expert accepts the invitation the remote station automatically starts receiving \& displaying the signal. At this time, only two participants may concurrently receive the ECG in real-time. There are plans to extend the real-time ECG component to support multicasting.

The operation of the electronic stethoscope in the context of the eHealth station is analogous to that of the cardiograph for real-time ECG acquisition as far as the interaction between the expert and the GP are concerned. The only difference is that in the context of auscultation, video conferencing is necessary to associate the auscultation sound with the position of the bell. For offline auscultation, the GP that conducts the examination needs to specify the exact position on the body where the stethoscope was placed.

The hand-held spirometer that has been integrated in the eHealth station is a store-and-forward device. The doctor conducts the examination under the guidance of the expert using video conferencing. Then, the spirometer is connected to the serial port and the examination is downloaded to the computer. A viewer has been developed to display graphically the examination results (see figure 5). Unfortunately, so far there is no widely accepted standard for the storage of spirometry data. 
Therefore, a proprietary format has been defined and the viewer supports it. When the user adds the examination to the current clinical document folder, the spirometry examination file is updated with the patient data. The lack of widely accepted standards in communication and storage of medical data is a considerable problem; thus, in the process of integrating different brands of devices, this development effort has to be repeated for every new model.

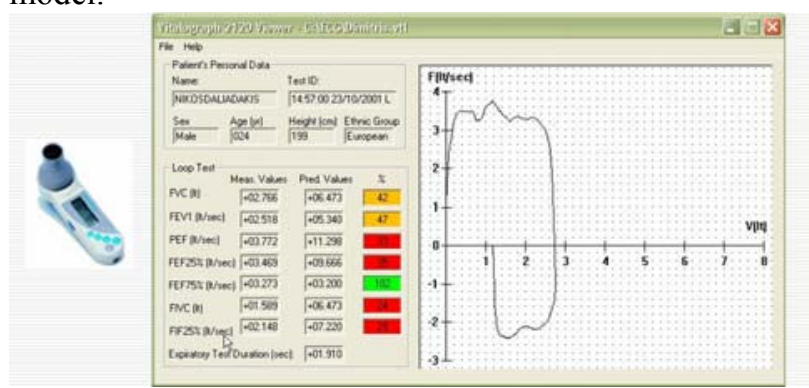

Figure 5. The handheld spirometer that has been integrated in the eHealth station and its viewer.

\section{2. eHealth service profiles}

One of the objectives of the eHealth platform is to facilitate the engineering of eHealth services tailored to specific medical disciplines and clinical problems. This can be partly accomplished through the definition of clinical documents conforming to the Clinical Document Architecture (CDA) of HL7 (an ANSI standard). The clinical documents, which consist of reusable XML fragments such as patient demographics, medical history, various observations, etc, can be employed as the means to incorporated clinical guidelines for eHealth services.

Furthermore, clinical documents in the CDA standard can be tied to terminology services and clinical information systems. In this way, based on the current clinical context, relevant medical data such as past examinations, can be automatically retrieved from the associated clinical information system or the integrated Electronic Health Record and be available as part of the shared clinical folder. This automatic retrieval of data helps in reducing error and eliminates the need of reentering medical data.

\subsection{Digital signing of clinical objects}

The eHealth services platform is taking advantage of the available public key infrastructure, to allow users of the eHealth station to electronically sign the clinical objects, they add to the shared clinical folder. Upon user registration, healthcare professionals receive a personal smart card that allows them to authenticate in the various clinical information systems and digitally sign diagnostic reports, referrals, or notes.

The electronic signature is stored in XML according to the W3C/IETF standard. Two types of electronic signature are supported by the eHealth platform: detached and enveloped. Detached signatures refer to any object through a universal resource locator (URI). Therefore, the signature is valid only in the context of the medical collaboration environment and verifies that the owner of the smart card actually entered the corresponding object in the shared clinical folder. The signature file includes the date of the signature, the certificate of the signer, a hash value for the object and the signature value itself to allow verification. Enveloped signatures are used only for clinical documents in the HL7/CDA standard. In that case the corresponding XML document includes an element that stores the signature. In all cases, to electronically sign a clinical object, one needs to enter his/her personal smart card, and type the pin number. This digital signing process protects from unauthorized use of the service and is accepted by national and European law.

\section{Conclusions \& future work}

An eHealth platform has been developed based on instant messaging and presence technology. Medical device components and clinical documents tied to clinical guidelines have been employed as part of eHealth service profiles. The overall architecture and service is extensible, customizable, and supports open interoperability standards. Healthcare professionals are attracted by the informal way of establishing communication and its advanced notification features.

The eHealth services platform is currently being validated in the regions of Crete and the south Aegean islands. Future work includes the definition and refinement of eHealth protocols, the integration of new devices and the refinement of existing medical device components. Furthermore, there are plans to make the eHealth station available to portable and mobile devices, by appropriately adjusting the user interface and the communication requirements of the individual components.

\section{References}

[1] Chronaki CE, Lees PJ, Antonakis N, Chiarugi F, Vrouchos $\mathrm{G}$, et al. Preliminary Results from the Deployment of Integrated Teleconsultation Services in Rural Crete. Computers in Cardiology 21, 2001;28:671-674.

[2] IETF working group on Instant Messaging and Presence Protocol (IMPP). http://www.ietf.org/html.charters/imppcharter.html.

[3] IETF working group on XML digital signatures. http://www.ietf.org/html.charters/xmldsig-charter.html.

Address for correspondence.

Catherine E. Chronaki

Center for Medical Informatics \&

Health Telematics applications

Institute of Computer Science - FORTH

PO 1385, 7110 Heraklion, Crete, Greece

Email: chronaki@ics.forth.gr 